\title{
Effect of a centralized prescription network on inappropriate prescriptions for opioid analgesics and benzodiazepines
}

\author{
Colin R. Dormuth ScD, Tarita A. Miller BSc, Anjie Huang MSc, Muhammad M. Mamdani PharmD MPH, \\ David N. Juurlink MD PhD; for the Canadian Drug Safety and Effectiveness Research Network
}

See related commentary by Wilsey and Prasad at www.cmaj.ca/lookup/doi/10.1503/cmaj.121495

Competing interests: Colin Dormuth's institution received a grant from the Canadian Institutes of Health Research through the Sunnybrook Research Institute. He has been reimbursed by the

Sunnybrook Research Institute for travel expenses. Until July 2011 , his faculty salary was partially paid through a contribution from the $\mathrm{BC}$ Ministry of Health, not specifically for this study. David Juurlink's institution received a grant from the Canadian Drug Safety and Effectiveness Research Network. Muhammad Mamdani has received consultancy fees from AstraZeneca, Bristol-Myers Squibb, Eli Lilly, GlaxoSmithKline, Hoffmann-La Roche, Novartis, Novo Nordisk and Pfizer. No other competing interests declared.

Members of the Canadian Drug Safety and Effectiveness Research Network appear at the end of the article.

This article has been peer reviewed.

Correspondence to: Colin R. Dormuth, colin.dormuth@ti.ubc.ca

CMAJ 2012. DOI:10.1503 /cmaj.120465

\begin{abstract}
Background: Opioid analgesics and benzodiazepines are often misused in clinical practice. We determined whether implementation of a centralized prescription network offering real-time access to patient-level data on filled prescriptions (PharmaNet) reduced the number of potentially inappropriate prescriptions for opioids and benzodiazepines.

Methods: We conducted a time series analysis using prescription records between Jan. 1, 1993, and Dec. 31, 1997, for residents of the province of British Columbia who were receiving social assistance or were 65 years or older.

pensed at a different pharmacy within 7 days after a filled prescription of at least 30 tablets of the same drug).

Results: Within 6 months after implementation of PharmaNet in July 1995, we observed a relative reduction in inappropriate filled prescriptions for opioids of $32.8 \%$ (95\% confidence interval $[\mathrm{Cl}] 31.0 \%-34.7 \%$ ) among patients receiving social assistance; inappropriate filled prescriptions for benzodiazepines decreased by $48.6 \%$ (95\% Cl 43.2\%-53.1\%). Similar and statistically significant reductions were observed among residents 65 years or older.
\end{abstract} We calculated monthly percentages of filled prescriptions for an opioid or a benzodiazepine that were deemed inappropriate (those issued by a different physician and dis-

$\mathrm{M}$ ost pharmacies and an increasing number of physicians rely on computer systems to maintain patient profiles that include clinical information, laboratory reports and medication records. ${ }^{1,2}$ Such systems provide a measure of safety with regard to medication errors, therapeutic duplication and drug interactions. ${ }^{3,4}$ However, they frequently operate in isolation and without information on prescriptions issued by other prescribers or dispensed at other pharmacies.

Centralized prescription networks may be a useful tool to reduce inappropriate prescribing and dispensing of prescriptions, particularly with controlled substances that are prone to misuse or resale, such as benzodiazepines and opioid analgesics. For example, a recent study found that almost 1 in 5 patients undergoing methadone maintenance therapy received and filled prescrip-
Interpretation: The implementation of a centralized prescription network was associated with a dramatic reduction in inappropriate filled prescriptions for opioids and benzodiazepines.

tions for other opioids, most often from a different physician or pharmacy.

Many jurisdictions throughout North America have implemented prescription monitoring programs that track the prescription and sale of controlled substances, ${ }^{6}$ and there is some evidence they are effective at reducing misuse of opioids.? However, these systems do not offer real-time information to pharmacists at the time of prescription processing, as a centralized prescription network would.

In July 1995, the province of British Columbia implemented a real-time processing system (PharmaNet) that links all BC pharmacies and hospitals to a centralized database containing real-time data on the prescription records of all provincial residents. ${ }^{8}$ In this study, we examined the impact of PharmaNet on inappropriate prescribing and dispensing of prescriptions for opioid analgesics and benzodiazepines. 


\section{Methods}

\section{Study design and datasets}

The intervention of interest was the populationwide implementation of PharmaNet in all community pharmacies in BC in July 1995. We used an interrupted time series analysis in which the proportion of all filled prescriptions per month that were deemed inappropriate (defined below) was regressed on time, using 30 months of data preceding the implementation of PharmaNet and 30 months of data thereafter. Prescriptions for the analysis were extracted from a BC Ministry of Health data warehouse that included prescription records from the PharmaNet database (all prescriptions filled by community pharmacies), as well as an earlier prescription database that included nearly all prescriptions for patients 65 years of age or older and patients receiving social assistance at the time their prescriptions were filled. The earlier database did not have real-time functionality, and pharmacists could not view prescriptions filled at other pharmacies. Also, the earlier database was missing most prescriptions for people not receiving social assistance and those less than 65 years old, which limited our analysis to the aforementioned groups.

\section{Inappropriate filled prescriptions}

The primary outcome was the proportion of filled prescriptions for a study drug deemed potentially inappropriate, defined in the following manner. First, we identified all filled prescriptions for 30 or more tablets of an opioid analgesic or a benzodiazepine during a 5-year period (Jan. 1, 1993, to Dec. 31, 1997). We chose this number of tablets because it is a commonly prescribed quantity that should, in many instances, encompass at least 7 days of treatment. For each filled prescription, patients were followed for 7 days to ascertain whether another prescription for the same drug was filled. We defined a subsequent filled prescription as inappropriate if it was issued by a different physician and dispensed at a different pharmacy. Then, we assembled 4 datasets representing 2 patient groups (seniors and recipients of income assistance) and 2 drug classes (opioids and benzodiazepines). Each dataset contained one observation per month, where each observation was the proportion of filled prescriptions that month that were identified as inappropriate according to our definition.

\section{Statistical analysis}

We used ordinary least-squares regression to estimate the impact of PharmaNet on the proportion of inappropriate filled prescriptions.
Regression models were estimated for each of the 4 datasets using the 30 months of available data preceding the implementation of PharmaNet in July 1995. The dependent variable in each model was the proportion of filled prescriptions deemed inappropriate. Independent variables in the models included time (in months), 11 monthly indicator variables for calendar month and autoregressive lag variables to compensate for temporal correlations.

We calculated the change in inappropriate filled prescriptions following the implementation of PharmaNet by summing the differences between observed and predicted monthly levels of inappropriate filled prescriptions across the 30 -month interval. Predicted values for the postPharmaNet period were generated from the linear regressions on pre-PharmaNet data. We calculated $95 \%$ confidence intervals (CIs) using an iterative process, replicated 1000 times, in which each observed value in the post-PharmaNet period was subtracted from a randomly generated value drawn from a normal distribution defined by the corresponding predicted value and its standard error. ${ }^{9}$

Finally, to test for specificity, we replicated our analysis using prescriptions for 3 commonly used nonsteroidal anti-inflammatory drugs (NSAIDs; oral formulations of ibuprofen, naproxen and diclofenac) to determine whether an impact of PharmaNet on filled prescriptions for opioids and benzodiazepines represented a more general effect on duplicate prescriptions, rather than an effect specific to drugs prone to abuse.

\section{Results}

\section{Residents receiving social assistance}

For residents receiving social assistance during the study period, we identified 1353118 filled prescriptions for opioids among 86704 patients (median age 40 [interquartile range (IQR) 3250] years) and 1483368 filled prescriptions for benzodiazepines among 47983 patients (median age 42 [IQR 35-51] years).

In the 30 months before the implementation of PharmaNet, $3.2 \%$ of opioid and $1.2 \%$ of benzodiazepine filled prescriptions on average were deemed inappropriate according to our definition. We observed a rapid and sustained decrease in the monthly proportion of inappropriate filled prescriptions for both drug classes after the implementation of PharmaNet (Figure 1). Overall, there was a $32.8 \%$ relative reduction in inappropriate prescriptions for opioids $(95 \%$ CI $31.0 \%-34.7 \%$ ) and a $48.6 \%$ relative reduction in inappropriate prescriptions for benzodiazepines (95\% CI $43.2 \%-53.1 \%$ ) in the first 30 months 
after PharmaNet was implemented. These estimated relative reductions for opioids and benzodiazepines translate to average absolute reductions of $1.08 \%$ and $0.49 \%$ per month, respectively.

\section{Senior residents}

For provincial residents 65 years or older, we identified 1180721 filled prescriptions for opioids among 199497 patients (median age 73 [IQR 69-78] years) and 2010548 benzodiazepine prescriptions among 150699 patients (median age 74 [IQR 69-79] years).

In the 30 months before PharmaNet implementation, $0.15 \%$ of filled prescriptions for opioids and $0.62 \%$ of filled prescriptions for benzodiazepines on average were found to be inappropriate. There was a $40.1 \%$ relative reduction in inappropriate prescriptions for opioids (95\% CI $32.0 \%-48.0 \%)$ and a $42.4 \%$ relative reduction in inappropriate prescriptions for benzodiazepines (95\% CI 31.4\%-53.2\%) in the first 30 months after PharmaNet was implemented (Figure 2). These estimated relative reductions for opioids and benzodiazepines translate to average absolute reductions of $0.28 \%$ and $0.05 \%$ per month, respectively.

As expected, we found that inappropriate filled prescriptions for NSAIDs were infrequent in both groups and did not change after the implementation of PharmaNet (Figure 3).

\section{Interpretation}

Our analysis showed that the implementation of a province-wide centralized prescription network was associated with large, immediate and sustained reductions in filled prescriptions for opioid analgesics and benzodiazepines deemed inappropriate by our definition. These findings provide empirical evidence that centralized prescription networks can reduce inappropriate prescribing and dispensing of prescriptions by offering health care professionals real-time access to prescription data. Physicians did not have access to PharmaNet when it was first introduced; consequently, the reductions observed in our study likely reflect the availability of real-time prescription information to front-line pharmacists.

Although the effectiveness of centralized systems may vary across jurisdictions, such differences are likely irrelevant from a policy standpoint, because even modest reductions in inappropriate prescriptions are clinically meaningful. If our findings can be generalized to other jurisdictions, we estimate that such networks could eliminate millions of inappropriate filled prescriptions in the United States and Canada annually.

\section{Limitations}

Some limitations of our study merit emphasis. Defining filled prescriptions as inappropriate

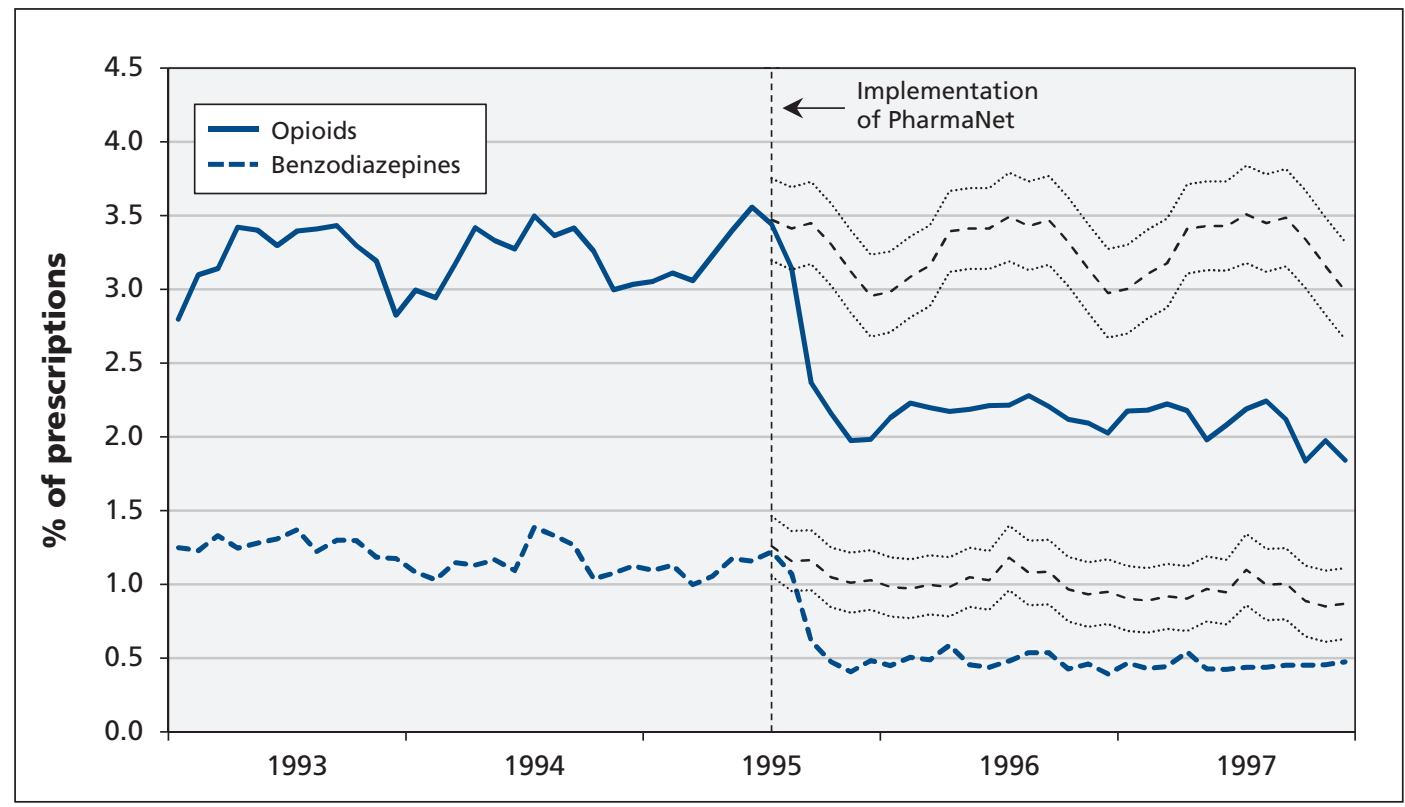

Figure 1: Monthly percentages of filled prescriptions for opioid analgesics and benzodiazepines deemed inappropriate among residents of British Columbia receiving social assistance before and after the implementation of PharmaNet, a centralized prescription network. The dashed and dotted lines shown after the implementation of PharmaNet represent the predicted percentages and their $95 \%$ confidence intervals had the system not been implemented. (The method for calculating the overall change in inappropriate filled prescriptions during the 30 -month period following implementation of the prescription network is described in the Methods section.) 
with the use of claims data is not straightforward. We purposefully employed a rigid definition so that our estimates of system impact would be conservative. Our definition may have resulted in some appropriate prescriptions being classified as inappropriate. However, it excluded many other inappropriate prescriptions, including those characterized by smaller quantities ( $<30$ tablets), longer intervals between prescriptions, and provenance from either a single prescriber or a single pharmacy. Our definition also excluded situations involving proximate pre- scriptions for different drugs, such as oxycodone and hydromorphone, and lorazepam and alprazolam. Consequently, our results almost certainly underestimate the ability of such networks to reduce inappropriate prescribing and dispensing of prescriptions. We used a 7-day window rather than a longer follow-up window to avoid classifying appropriate prescriptions as inappropriate. Inherent to this choice was an assumption that the probability of accurately identifying an inappropriate prescription decreased as more time elapsed between prescriptions.

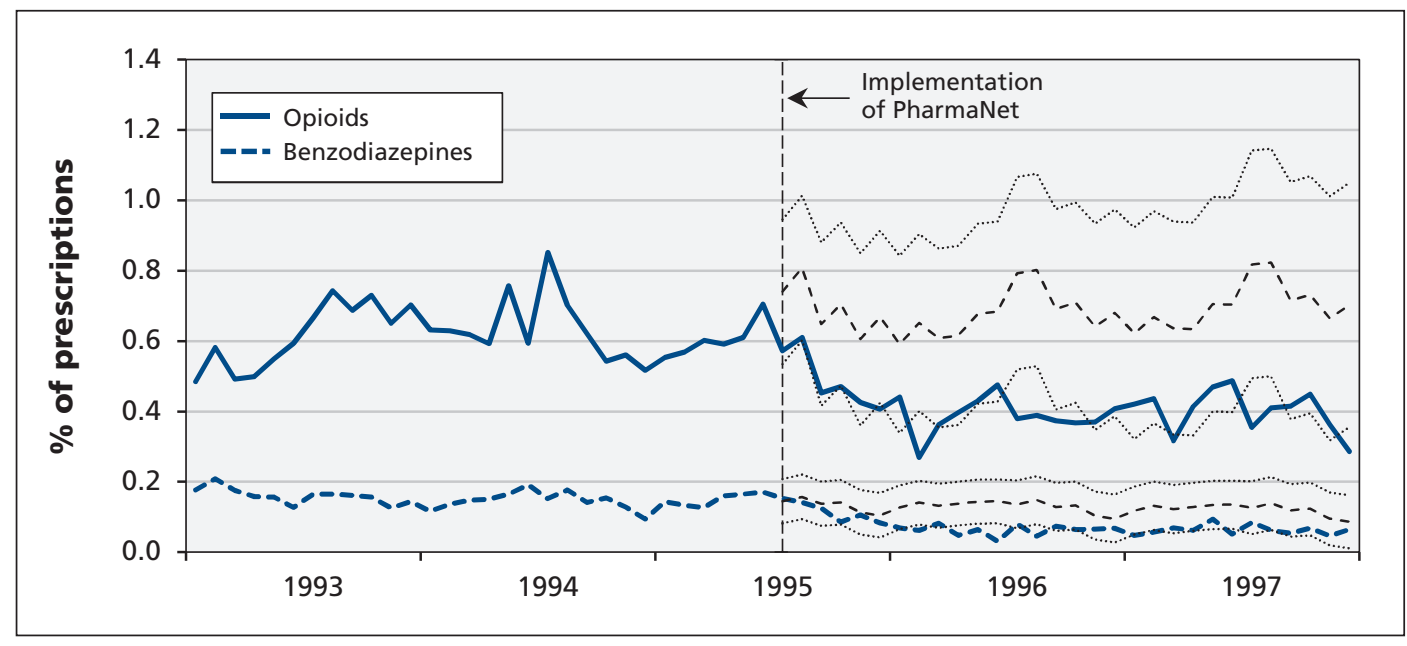

Figure 2: Monthly percentages of filled prescriptions for opioids and benzodiazepines deemed inappropriate among residents of British Columbia 65 years of age or older before and after the implementation of PharmaNet. The dashed and dotted lines shown after the implementation of PharmaNet represent the predicted percentages and their $95 \%$ confidence intervals had the system not been implemented. (The method for calculating the overall change in inappropriate filled prescriptions during the 30-month period following implementation of the prescription network is described in the Methods section.)



Figure 3: Monthly percentages of filled prescriptions for nonsteroidal anti-inflammatory drugs deemed inappropriate among residents of British Columbia 65 years of age or older and residents receiving social assistance during the study period. 
Some pharmacies started using PharmaNet before the summer of 1995, when most pharmacies went online. This limitation could only have attenuated any observed effect of PharmaNet.

The effect of PharmaNet on benzodiazepine prescriptions may have been augmented by an educational letter on sleep management mailed to physicians and pharmacists in BC in January 1996. ${ }^{10}$ However, a previous evaluation of the letter suggests it was associated with a nonsignificant reduction in de novo prescribing of benzodiazepines, ${ }^{11}$ and no such letter was issued for opioids.

Finally, our analysis reflects data from a natural experiment that took place 17 years ago. However, the data are of high quality and are particularly relevant in light of prevailing concerns regarding the abuse and diversion of prescription drugs. ${ }^{12}$

\section{Conclusion}

The implementation of a centralized prescription network was associated with a dramatic reduction in potentially inappropriate filled prescriptions for opioids and benzodiazepines. We speculate that wider implementation of such networks could substantially reduce the costs and harms associated with misuse of prescription drugs.

\section{References}

1. Hsiao CJ, Burt CW, Rechtsteiner E, et al. Preliminary estimates of electronic medical records use by office-based physicians: United States, 2008. Natl Health Stat Report 2008. Available: www.cdc.gov/nchs/data/hestat/physicians08/physicians08.htm (accessed 2012 Feb. 2).

2. Hing E, Hall MJ, Ashman JJ. Use of electronic medical records by ambulatory care providers: United States, 2006. Natl Health Stat Report 2010;Apr. 30:1-21.

3. Appari A, Carian EK, Johnson ME, et al. Medication administration quality and health information technology: a national study of US hospitals. J Am Med Inform Assoc 2012;19:360-7.

4. Aspden P, Wolcott JA, Bootman JL, et al., editors. Preventing medication errors. Washington (DC): National Academy Press; 2007. p. 409-46.

5. Kurdyak P, Gomes T, Yao Z, et al. Use of other opioids during methadone therapy: a population-based study. Addiction 2012; 107:776-80.

6. Simeone R, Holland L. Executive summary: an evaluation of prescription drug monitoring programs. Albany (NY): Simeone Associates; 2006. Available: www.namsdl.org/resources /PDMP\%20Study\%20Executive\%20Summary.pdf (accessed 2012 Feb. 28).

7. Reifler LM, Droz D, Bailey JE, et al. Do prescription monitoring programs impact state trends in opioid abuse/misuse? Pain Med 2012;13:434-42.
8. PharmaNet. Vancouver (BC): BC Ministry of Health Services; 2011. Available: www.health.gov.bc.ca/pharmacare/pharmanet /netindex.html (accessed 2012 Feb. 28).

9. Dormuth CR, Glynn RJ, Neumann P, et al. Impact of two sequential drug cost-sharing policies on the use of inhaled medications in older patients with chronic obstructive pulmonary disease or asthma. Clin Ther 2006;28:964-78.

10. Therapeutics initiative. To sleep or not to sleep: Here are your questions. Therapeutics Letter 1995;11: Nov./Dec.

11. Dormuth CR, Maclure M, Bassett K, et al. Effect of periodic letters on evidence-based drug therapy on prescribing behaviour: a randomized trial. CMAJ 2004;171:1057-61.

12. Dhalla IA, Persaud N, Juurlink DN. Facing up to the prescription opioid crisis. BMJ 2011;343:d5142.

Affiliations: From the Department of Anesthesiology, Pharmacology and Therapeutics (Dormuth), University of British Columbia, Vancouver, BC; the Therapeutics Initiative (Dormuth, Miller), University of British Columbia, Vancouver, $\mathrm{BC}$; the Institute for Clinical Evaluative Sciences (Huang, Mamdani, Juurlink), Toronto, Ont.; the Institute of Health Policy, Management and Evaluation (Mamdani), University of Toronto, Toronto, Ont.; the Li Ka Shing Knowledge Institute of St. Michael's Hospital (Mamdani), Toronto, Ont.; the Sunnybrook Research Institute (Juurlink), Sunnybrook Health Sciences Centre, Toronto, Ont.; and the Departments of Medicine and Pediatrics (Juurlink), University of Toronto, Toronto, Ont.

Contributors: Colin Dormuth, Muhammad Mamdani and David Juurlink conceived of the study design and drafted the manuscript. Colin Dormuth, Tarita Miller and Anjie Huang analyzed the data. All of the authors revised the article critically for important intellectual content and approved the final version submitted for publication.

Funding: This study was funded by a grant from the Canadian Institutes of Health Research (CIHR) and by a contribution from the BC Ministry of Health to the University of British Columbia. The Canadian Drug Safety and Effectiveness Research Network is funded by an Emerging Team Grant from the CIHR (grant no. ETG-92247). The CIHR had no role in the study design; the collection, analysis or interpretation of the data; the writing of the report; or the decision to submit the article for publication.

Acknowledgement: The authors thank Irfan Dhalla for comments on an earlier version of this manuscript.

Members of the Canadian Drug Safety and Effectiveness Research Network: David N. Juurlink (chair), Institute for Clinical Evaluative Sciences, Toronto, Ont.; Colin R. Dormuth, University of British Columbia, Vancouver, BC; Chelsea Hellings (program coordinator), Institute for Clinical Evaluative Sciences, Toronto, Ont.; Anita L. Kozyrskyj, University of Manitoba, Winnipeg, Man.; Muhammad M. Mamdani, St. Michael's Hospital, Toronto, Ont.; Yola Moride, Université de Montréal, Montréal, Que.; J. Michael Paterson, Institute for Clinical Evaluative Sciences, Toronto, Ont.; Colette Raymond, Manitoba Centre for Health Policy, University of Manitoba, Winnipeg, Man.; and Robyn Tamblyn, McGill University, Montréal, Que. 